\title{
Interplanetary magnetic field orientation and the magnetospheres of close-in exoplanets
}

\author{
E. P. G. Johansson ${ }^{1}$, J. Mueller ${ }^{2}$, and U. Motschmann ${ }^{2,3}$ \\ ${ }^{1}$ Astrophysikalisches Institut Potsdam, An der Sternwarte 16, 14482 Potsdam, Germany \\ e-mail: ejohansson@aip.de \\ 2 Institute for Theoretical Physics, TU Braunschweig, Mendelssohnstrasse 3, 38106 Braunschweig, Germany \\ e-mail: [joa.mueller;u.motschmann] @tu-bs.de \\ 3 Institute for Planetary Research, DLR, Berlin, Germany
}

Received 15 April 2010 / Accepted 26 July 2010

\begin{abstract}
The abundance of exoplanets with orbits smaller than that of Mercury most likely implies that there are exoplanets exposed to a quasiparallel stellar-wind magnetic field. Many of the generic features of stellar-wind interaction depend on the existence of a nonzero perpendicular interplanetary magnetic field component. However, for closer orbits the perpendicular component becomes smaller and smaller. The resulting quasiparallel interplanetary magnetic field may imply new types of magnetospheres and interactions not seen in the solar system. We simulate the Venus-like interaction between a supersonic stellar wind and an Earth-sized, unmagnetized terrestrial planet with ionosphere, orbiting a Sun-like star at 0.2 AU. The importance of a quasiparallel stellar-wind interaction is then studied by comparing three simulation runs with different angles between stellar wind direction and interplanetary magnetic field. The plasma simulation code is a hybrid code, representing ions as particles and electrons as a massless, charge-neutralizing adiabatic fluid. Apart from being able to observe generic features of supersonic stellar-wind interaction we observe the following changes and trends when reducing the angle between stellar wind and interplanetary magnetic field 1) that a large part of the bow shock is replaced by an unstable quasiparallel bow shock; 2) weakening magnetic draping and pile-up; 3) the creation of a second, flanking current sheet due to the need for the interplanetary magnetic field lines to connect to almost antiparallel draped field lines; 4) stellar wind reaching deeper into the dayside ionosphere; and 5) a decreasing ionospheric mass loss. The speed of the last two trends seems to accelerate at low angles.
\end{abstract}

Key words. plasmas - planetary systems - stars: winds, outflows - magnetic fields

\section{Introduction}

The discovery of exoplanets has provided a plethora of examples of planets both different and extremely different from those in our solar system. Among the most interesting planets from a stellar wind interaction point of view are those with semimajor axes smaller than Mercury's perihelion (0.31 AU) and thus without counterpart in the solar system. At least $37 \%$ of the presently known exoplanets fall within this category ${ }^{1}$ including the more famous so-called close-in extrasolar giant planets (cEGPs), also known as "Hot Jupiters", with semimajor axes of $r<0.05-0.1$ AU.

The small star-planet separation has many potential implications for stellar wind interaction. To begin with, we can expect different stellar wind parameters, in particular in the form of a more dense wind with stronger magnetic fields. We can also expect stronger photoionization that produces more ionospheric plasma, which in the case of an absent intrinsic magnetic field can interact with the stronger stellar wind. Less obvious scenarios, which both may and may not occur and are all unrealized in today's solar system are: 1) subsonic stellar wind interaction at sufficiently small distances (Ip et al. 2004; Preusse et al. 2005) possibly enabling information to travel from the planet to the star via the stellar wind (see e.g. Lanza 2009, and references therein);2) the possibility of stellar wind interaction with hydrodynamically expanding atmospheres, a type

\footnotetext{
${ }^{1}$ Retrieved on April 15th 2010 from The Extrasolar Planets Encyclopaedia at http://exoplanet.eu/.
}

of atmosphere caused by extreme heating that reaches altitudes of $\sim R_{\mathrm{p}}$ and continuously loses material into space (see e.g. Johansson et al. 2009; Vidal-Madjar et al. 2003, 2004, 2008; Lammer et al. 2004; Chamberlain \& Hunten 1987); 3) the possibility of detectable stellar-wind-powered low-frequency emissions from cEGPs similar to that from the magnetized planets in the solar system but enhanced by the stronger stellar wind (see e.g. Griessmeier et al. 2007a,b; Lazio et al. 2004; Farrell et al. 1999; Zarka et al. 2001); and 4) a range of orbital distances with quasiparallel interaction, i.e. stellar wind interaction where the interplanetary magnetic field (IMF) is approximately parallel to the stellar wind velocity in the frame of the planet.

Among the known exoplanets within the orbit of Mercury, giants do dominate but this is very likely to be an observational effect. We speculate, based on theory and simulations of planet formation and the statistics of known exoplanets (Fogg \& Nelson 2009; Raymond et al. 2006; Lin 2006), that there are also terrestrial exoplanets yet to be discovered within this orbital regime. New space missions such as CoRoT, and in particular Kepler, are rapidly taking us toward the point where we can detect and investigate not only terrestrial, but even Earth-sized close-in exoplanets (Bordé et al. 2003; Gaidos et al. 2007; Borucki et al. 2010; Lawson et al. 2004).

We previously studied one of the abovementioned scenarios in Johansson et al. (2009) that used plasma simulations to study the stellar wind interaction with an extreme version of a hydrodynamically expanding atmosphere belonging to an unmagnetized, terrestrial planet. This work continues by studying 


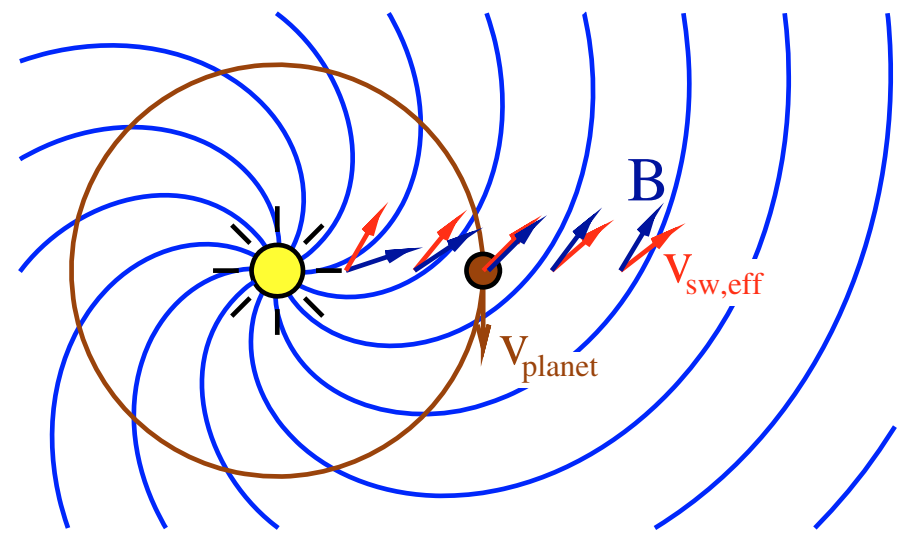

Fig. 1. Illustration of how the direction of the IMF and the effective stellar change with distance from the star. $\boldsymbol{v}_{\text {planet }}$ (brown) is the orbital velocity of the planet, $\boldsymbol{v}_{\mathrm{sw}, \mathrm{eff}}$ (red) is the effective stellar wind direction in the frame of a planet in circular orbit and $\boldsymbol{B}$ (blue) is the IMF direction. The blue spiral lines represent Parker spirals (magnetic field lines). The depicted planet is in an orbit where effective stellar wind and magnetic field are parallel.

quasiparallel stellar-wind interaction with a Venus-like exoplanet using simulations. Both Johansson et al. (2009) and this work make use of hybrid simulations, a plasma simulation model that describes ions as particles and electrons as a massless, charge-neutralizing adiabatic fluid. Compared to pure fluid models, e.g., magnetohydrodynamics (MHD), this has the advantage of being able to handle non-Maxwellian ion distributions and resolve kinetic effects, e.g. pick-up.

\section{Quasiparallel stellar wind interaction}

To understand why we should expect quasiparallel interaction, we must first consider the geometry of the IMF in the solar system and which we assume is duplicated in other star systems. In short, magnetic field lines are frozen into the stellar wind and are thus carried away from the star in the radial direction. However, the bases of the very same field lines are frozen into the star and therefore follow its rotation. This configuration leads to the magnetic field lines in the ecliptic plane forming approximate Archimedean spirals, or Parker spirals. A consequence of this shape is a magnetic field that is azimuthal far away from the star (e.g. at Pluto in the solar system) and becomes radial at smaller distances $^{2}$. An object in circular orbit however, experiences a stellar wind velocity that is radial in orbits far away from the star but azimuthal in lower orbits, due to increasing orbital velocity. It follows from this that there should be a certain distance where an object in circular orbit with velocity $v_{\text {planet }}$ should ideally experience an IMF that is always parallel to the stellar wind direction. This is illustrated in Fig. 1.

To approximately calculate this distance where the IMF is aligned to the stellar wind velocity we make use of the simple observation that a planet in such an orbit must always lie on the very same spiral-shaped magnetic field line as the field line rotates around the star. Therefore the orbital period must coincide with the stellar rotation period, i.e.,

$\Omega_{\star} r=\sqrt{\frac{G M_{\star}}{r}}$,

\footnotetext{
2 This argument technically assumes that the stellar wind speed does not increase too rapidly with distance.
}

where $\Omega_{\star}$ is the stellar angular rotation velocity, $M_{\star}$ is the stellar mass, $G$ is the gravitational constant, and $r$ is the orbital distance. This can be rewritten as

$r=\left(G M_{\star}\right)^{1 / 3} \Omega_{\star}^{-2 / 3}$,

which for ease of use can also be rephrased as

$r \approx 0.020 \times\left(\frac{M_{\star}}{M_{\odot}}\right)^{1 / 3}\left(\frac{T_{\star}}{1 \text { day }}\right)^{2 / 3} \mathrm{AU}$

where $M_{\odot}$ is the solar mass and $T_{\star}$ is the stellar rotation period. Using for example solar values infers $r=0.16 \mathrm{AU}$ or about half of Mercury's perihelion distance of $0.31 \mathrm{AU}$. We note that the above expression is conveniently independent of stellar wind velocity and really only depends on the IMF being frozen-in. It does not assume that the stellar wind velocity is constant over different radial distances. We have of course also made use of an ideal picture, neglecting time-varying stellar wind velocity, turbulence, shock waves etc. A planet in the orbit described above is thus more likely to be exposed to a stellar wind that is parallel in some average sense rather exactly parallel at every point in time. This combined with the improbability of finding exoplanets in exactly these orbits is the reason why we prefer to speak of quasiparallel stellar wind interaction rather than parallel interaction.

The existence of orbits with quasiparallel stellar-wind interaction, i.e. where the IMF is approximately parallel to the stellar wind direction in the planet frame, is interesting because many of the generic features of stellar wind interaction depend on the existence of a perpendicular magnetic field component. Thus, planets in these orbits may display very different types of magnetospheres from what we are used to. Maybe the most obvious example of the dependence on a perpendicular magnetic field component is the draping of the magnetic field but also that pickup requires it, i.e. the removal of ionospheric ions through the stellar wind induced electric field.

The behavior of plasma shocks depends on the shock angle $\theta_{B n}$, i.e. the angle between the shock surface normal and the upstream magnetic field and is, as for stellar wind interactions as a whole, classified as either quasiperpendicular or quasiparallel depending on this angle (Baumjohann \& Treumann 1996). Since the bow shock around a planet is a more or less parabolic surface, only parts of the shock surface can be regarded as a quasiparallel shock for a quasiparallel stellar wind. For the same reason, a quasiparallel shock is also often present for quasiperpendicular stellar wind interactions but far out on the flanks where they have little impact.

Parallel and quasiparallel shocks are interesting because it can be shown that they are not stable and do not form welldefined shock surfaces the way we are used to seeing for quasiperpendicular shocks (Treumann \& Jaroschek 2008a,b). It can for example be shown that MHD shocks in theory reduce to gasdynamic shocks while in practice they are oscillatory up to large distances upstream (Baumjohann \& Treumann 1996). Hybrid simulations have also shown this to be true for high Mach numbers (Burgess 1989). This is because particles reflected from quasiparallel shocks can travel significant distances upstream leading to beam instabilities and an extended foreshock region (Treumann \& Jaroschek 2008a,b). This means that kinetic effects are important for quasiparallel shocks and hybrid simulations should therefore be better suited to the task than MHD. We do not delve into the theory of quasiparallel shocks but refer to Treumann \& Jaroschek (2008a,b) for more technical details. 
Although quasiparallel shocks have been thoroughly studied with hybrid simulations (see Filippychev 2000, and references therein), quasiparallel stellar wind interactions have not as one could expect given the absence of it in the solar system. In the light of this and the multitude of discovered exoplanets in recent years, we choose to study quasiparallel stellar wind interaction using a series of similarly configured hybrid simulation runs.

\section{The hybrid simulation model}

We used the "Adaptive Ion Kinetic Electron Fluid" (AIKEF) code for the simulation runs in this work. This code is essentially an improved successor of our previous hybrid simulation code introduced in Bagdonat \& Motschmann (2002). AIKEF uses the same physical model and numerical algorithms but has some additional features that were not used in this work. We therefore do not describe AIKEF to any great length but instead refer to Johansson et al. (2009) and Bagdonat \& Motschmann (2002) and references therein for details.

The basic purpose of the simulation code is to integrate the time evolution of collisionless plasmas in a three-dimensional simulation box. A planet is placed inside the simulation box and is exposed to a moving stellar wind plasma. The presence of an ionosphere is emulated by surrounding the planet with an additional thin plasma-producing layer. The simulation is then allowed to run until a quasistationary state has been reached.

Plasma ions are, on the one hand, modeled as kinetic superparticles, each representing the motion of a larger number of physical ions belonging to the same species. Electrons are, on the other hand, modeled as a massless, charge-neutralizing adiabatic electron fluid, hence the name "hybrid simulations". By assuming in particular a vanishing electron mass, quasineutrality, and Ampère's law with the Darwin approximation, we can describe the system unambiguously by the magnetic field and the positions and velocities of the superparticles (ions). In the process, we obtain the following equations that can then be numerically integrated over time. We have the time evolution of superparticles

$\frac{\mathrm{d} \boldsymbol{v}_{p}}{\mathrm{~d} t}=\frac{q_{p}}{m_{p}}\left(\boldsymbol{E}+\boldsymbol{v}_{p} \times \boldsymbol{B}\right)$,

where $\boldsymbol{v}_{p}, q_{p}$, and $m_{p}$ are the velocity, charge, and mass of superparticle $p, \boldsymbol{E}$ is the electric field, and $\boldsymbol{B}$ is the magnetic field. The time evolution of the magnetic field is described by

$\frac{\partial \boldsymbol{B}}{\partial t}=\nabla \times\left(\boldsymbol{u}_{\mathrm{i}} \times \boldsymbol{B}\right)-\nabla \times\left(\frac{\boldsymbol{j} \times \boldsymbol{B}}{e n_{\mathrm{i}}}\right)$,

where $\boldsymbol{u}_{\mathrm{i}}$ is the total ion bulk flow velocity, $\boldsymbol{j}$ is the total current, $e$ is the elementary charge, and $n_{\mathrm{i}}$ is the total ion density. The two above equations use expressions for the current

$\boldsymbol{j}=\frac{\nabla \times \boldsymbol{B}}{\mu_{0}}$

and the electric field

$\boldsymbol{E}=-\left(\boldsymbol{u}_{\mathrm{i}} \times \boldsymbol{B}\right)+\frac{\boldsymbol{j} \times \boldsymbol{B}}{e n_{\mathrm{i}}}-\frac{\nabla p_{\mathrm{e}}}{e n_{\mathrm{i}}}$,

where $\mu_{0}$ is the vacuum permeability and $p_{\mathrm{e}}$ is the total thermal electron pressure. The parameter $p_{\mathrm{e}}$ is calculated as the sum of partial electron pressures $p_{\mathrm{e}, s} \propto n_{\mathrm{i}, s}{ }^{2}$ for every ion species $s$, where $n_{\mathrm{i}, s}$ is the corresponding ion density.

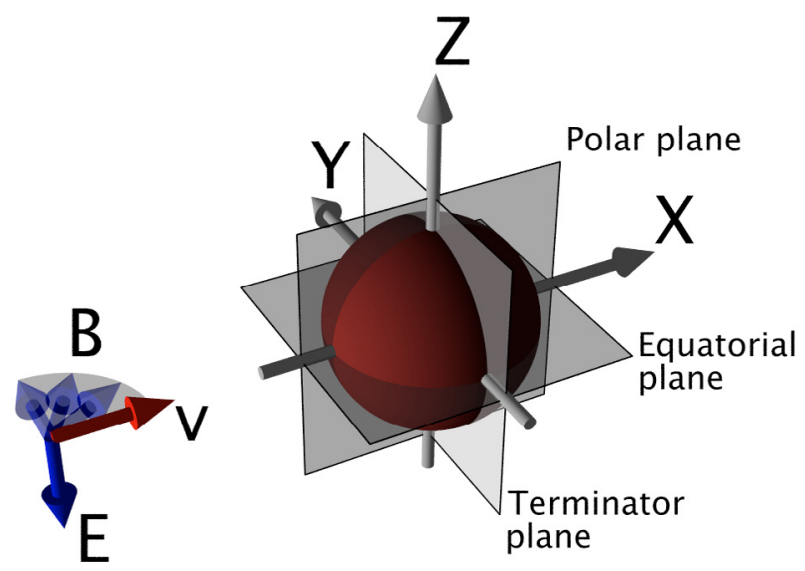

Fig. 2. Orientation of the coordinate axes relative to the stellar wind and IMF. The variables $v, B$, and $E$ refer to the stellar wind velocity, IMF, and electric field respectively of the undisturbed stellar wind, i.e. before the stellar wind interacts with the planet. The equatorial plane refers to the $x y$ plane, the polar plane refers to the $x z$ plane, and the terminator plane refers to the $y z$ plane.

Although the hybrid simulation paradigm is better suited than MHD to studying quasiparallel interaction, in particular the quasiparallel shock, it is still not perfect. Since it models electrons as an adiabatic fluid it cannot include any electron entropy increase over the bow shock.

\subsection{Coordinate system and naming conventions}

We follow the convention that the undisturbed stellar wind always travels in the positive $x$ direction and that the undisturbed IMF always lies in the $x y$ plane with non-negative $x$ and $y$ components. The convective electric field $\boldsymbol{E}=-\boldsymbol{v}_{\mathrm{sw}, 0} \times \boldsymbol{B}$, where $\boldsymbol{v}_{\mathrm{sw}, 0}$ is the stellar wind velocity vector, will consequently always be in the negative $z$ direction. Since both the IMF vector and the stellar wind vector lie approximately in the ecliptic plane, which in turn approximates the equatorial plane for most of the solar system planets, we refer to the $x y$ plane as the equatorial plane, although our simulated planet does not really rotate. The north pole and south pole are then where the $z$ axis crosses the planetary surface, at $z=+R_{\mathrm{p}}$ and $z=-R_{\mathrm{p}}$, respectively. Following a similar logic, we choose to refer to the $x z$ plane as the polar plane and the $y z$ plane as the terminator plane. Most of these conventions are illustrated in Fig. 2.

\section{Model parameters}

We chose not to consider a perfectly parallel stellar-wind interaction because 1) a truly parallel stellar wind interaction does not persist for very long due to the fluctuations of the stellar wind; 2 ) it is our experience that these simulations take a long time to reach quasistationary state; and 3) the simulated ionospheres appear to have difficulties in preventing the parallel stellar wind from penetrating. We wish to avoid this penetration because, among other things, it may lead to chemical reactions with the atmosphere that are not incorporated into the simulation model. We also do not wish to consider a single quasiparallel interaction in isolation but find it more useful to be able to compare it with a perpendicular interaction. This highlights the differences and unique features caused by the quasiparallel interaction rather than any peculiarities that might arise from our particular choice of parameters. An intermediate run has also been added so that we can study the transition between the two better. Having this 
range of simulations also has the additional advantage that one can more easily compare with simulations in the literature, e.g. Venus studies such as Martinecz et al. (2009) and Kallio et al. (2006).

Thus, we chose to perform three simulation runs, all of them identical with the exception of the angle $\alpha_{\mathrm{sw}, 0}$ between the undisturbed stellar wind velocity and the IMF. We refer to these runs as the perpendicular run $\left(\alpha_{\mathrm{sw}, 0}=90^{\circ}\right)$, the intermediate run $\left(\alpha_{\mathrm{sw}, 0}=30^{\circ}\right)$, and the quasiparallel run $\left(\alpha_{\mathrm{sw}, 0}=10^{\circ}\right)$.

Using a perfectly perpendicular simulation run as a reference is interesting not only because it is the very opposite of a parallel run, but also because it can be shown that it should ideally have a kind of mirror symmetry with the polar plane $(y=0)$ as a symmetry plane, i.e. $y>0$ and $y<0$ are mirror images of each other when using our simulation model. The equatorial plane $(z=0)$ is however not a symmetry plane but would have been had we used ideal or resistive MHD.

We chose to consider a Venus-like stellar wind interaction, i.e. an interaction between a supersonic stellar wind and an unmagnetized terrestrial planet with an atmosphere. Part of the reason for this is that we have little knowledge of the intrinsic magnetic fields to expect and wish to avoid having stronger magnetic fields since particle gyroradii and gyration times then decrease, pushing the numerical cost beyond a presently acceptable limit. For this purpose, we use an Earth-sized terrestrial exoplanet, $R_{\mathrm{p}}=R_{\text {Earth }}$, equipped with a Venus-inspired ionosphere at an orbital distance of $r=0.2$ AU from a Sun-like star as the basic scenario for our simulation runs. This distance is close to our previously estimated $r=0.16$ AU for a circular orbit around the Sun in which one would ideally experience a parallel stellar wind.

We used a simulation box with of size $5 \times 6 \times 7 R_{\mathrm{p}}$ divided into a grid of $104 \times 128 \times 148$ approximately cube-shaped cells. The exoplanet is located $-0.5 R_{\mathrm{p}}$ in the negative $z$ direction from the center of the box. An asymmetric box and the location of the planet were chosen to help us minimize the influence of wave reflections on the outer simulation box boundaries.

The simulation runs were all performed for a simulated time $t_{\text {sim }}$ equivalent to an undisturbed stellar wind passing through the box between 222 and 275 times, i.e. $\left(t_{\text {sim }} v_{\text {sw }, 0}\right) / L_{x}=222-275$, where $v_{\mathrm{sw}, 0}$ is the stellar wind velocity and $L_{x}$ is the length of the simulation box in the $x$ direction. These run times are in practice much longer than necessary to reach a quasistationary state, but are needed to obtain average values of the stellar wind lost to the planet and the ionospheric plasma lost from the planet.

\subsection{Stellar wind parameters}

We estimate the velocity, density, and ion and electron temperature of a hydrogen-only stellar wind at a distance of $0.2 \mathrm{AU}$ from a Sun-like star by following the same procedure as in Johansson et al. (2009). Applying a hybrid simulation code to a planet-sized obstacle does however constrain the maximum strength of the magnetic field that it is practically possible to work with due to the need to resolve every ion gyration into several time steps. We therefore work in a low-magnetic field limit using an IMF field strength of $12 \mathrm{nT}$. All stellar wind parameters and resulting Mach numbers are summarized in Table 1.

\subsection{Ionospheric parameters}

The ionosphere is modeled as an oxygen-ion-producing layer above a certain altitude where the atmosphere can be considered
Table 1. Stellar wind parameters.

\begin{tabular}{|c|c|c|}
\hline Parameter & Symbol & Value \\
\hline Velocity & $v_{\mathrm{sw}, 0}$ & $330 \mathrm{~km} \mathrm{~s}^{-1}$ \\
\hline density & & $210 \mathrm{~cm}^{-3}$ \\
\hline $\mathrm{IMF} /$ magnetic field & $B_{\mathrm{sw}, 0}$ & $12 \mathrm{nT}$ \\
\hline \multirow{3}{*}{$\begin{array}{r}\text { Velocity-IMF angle, } \\
\text { intermendicular run } \\
\text { quasiparallel run }\end{array}$} & $\alpha_{\mathrm{sw}, 0}$ & $90^{\circ}$ \\
\hline & $\alpha_{\mathrm{sw}, 0}$ & $30^{\circ}$ \\
\hline & $\alpha_{\mathrm{sw}, 0}$ & $10^{\circ}$ \\
\hline Ion temperature & $T_{\mathrm{sw}, \mathrm{i}, 0}$ & $154500 \mathrm{~K}$ \\
\hline Electron temperature & $T_{\mathrm{sw}, \mathrm{e}, 0}$ & $309000 \mathrm{~K}$ \\
\hline Alfvénic Mach number & $M_{\mathrm{A}}$ & 18 \\
\hline Magnetosonic Mach number & $M_{\mathrm{ms}}$ & 3.7 \\
\hline
\end{tabular}

collisionless. We then assume that ions that travel below this altitude are "recombined" in the denser atmosphere below. What happens below this altitude is thus of no concern and we therefore in effect allow the simulation code to use this absorbing boundary as the surface of the planet.

Mostly inspired by the upper atmosphere of Venus, we use an ionospheric production rate profile based on the photoionization of atomic oxygen, assuming that the neutral atomic oxygen has an ordinary hydrostatic profile

$n_{\mathrm{O}}(h)=n_{\mathrm{O}, \text { surf }} \mathrm{e}^{-h / H}$,

where $h$ is the altitude above the absorbing boundary, $n_{\mathrm{O} \text {, surf }}$ is the density at the absorbing boundary, $H$ is a constant scale height

$H=\frac{k_{\mathrm{B}} T_{\mathrm{O}}}{16 m_{\mathrm{p}} g} \approx 92.8 \mathrm{~km}=0.015 R_{\mathrm{p}}$

calculated using a neutral oxygen temperature $T_{\mathrm{O}}=1764 \mathrm{~K}$ and a gravitational acceleration of $g=9.81 \mathrm{~m} \mathrm{~s}^{-2}, k_{\mathrm{B}}$ is the Boltzmann constant and $m_{\mathrm{p}}$ is the proton mass. The neutral atmospheric temperature $T_{\mathrm{O}}$ is chosen to be higher than the temperature of the upper ionosphere and exosphere of Earth, 700-1100 K (Schunk \& Nagy 2000), or the exosphere of Venus, 200-1000 K (Chamberlain \& Hunten 1987) since we are considering a planet at a distance of only $r=0.2 \mathrm{AU}$ from a Sun-like star where more energy is available to heat the upper atmosphere. With this atmospheric profile, we justify the existence of a dayside ionospheric production rate that, using standard methods, takes the form of a Chapman profile given by

$Q_{\mathrm{O}^{+}}(h, \theta)=\left(n_{\mathrm{O}, \text { surf }} \mathrm{e}^{-h / H}\right) \sigma_{\mathrm{i}} I_{\infty} \exp \left(-\frac{\sigma_{\mathrm{a}} n_{\mathrm{O}, \text { surf }} H}{\cos \theta} \mathrm{e}^{-h / H}\right)$,

where $\sigma_{\mathrm{i}}$ and $\sigma_{\mathrm{a}}$ are the photoionization and absorption crosssections for atomic oxygen, $I_{\infty}$ is the photon flux, and $\theta$ is the angle of incidence of the incoming EUV radiation. We used this profile for $0^{\circ} \leq \theta \leq 87^{\circ}$, i.e. most of the dayside but still avoiding the singularities at the terminator. The cross-sections are photon flux-weighted average values from the EUVAC model (Schunk \& Nagy 2000; Richards et al. 1994). The photon flux is twice that of the EUVAC model scaled to $r=0.2 \mathrm{AU}$. The extra factor of two is, once again, to ensure that we stay in a regime where the ionosphere is strong enough to prevent the stellar wind from penetrating the ionosphere. While this extra factor is arbitrary, the difference is still no greater than that between the quiet and active Sun (Huebner et al. 1992).

We also add, somewhat arbitrarily, a small constant background production rate to compensate for there being ionization mechanisms other than photoionization and to prevent Eqs. (5) 
E. P. G. Johansson et al.: IMF orientation and the magnetospheres of close-in exoplanets

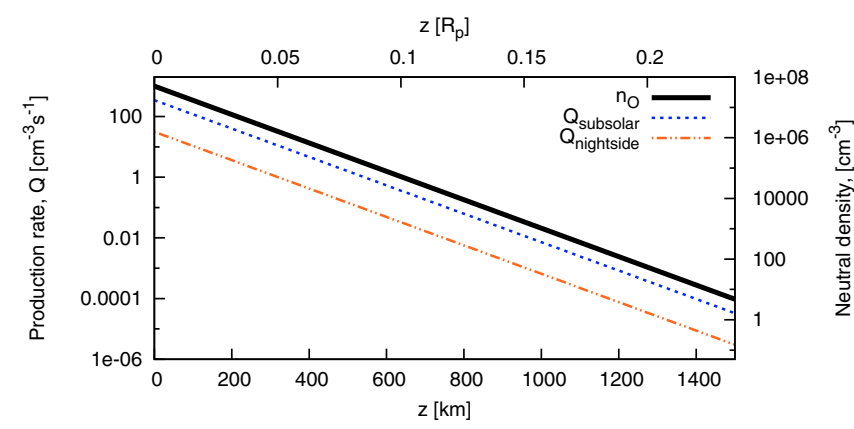

Fig. 3. Ionospheric profiles used in simulation runs. Black solid line: neutral atomic oxygen density (right-hand $y$ scale). Blue dotted line: substellar ionospheric production rate (left-hand $y$ scale). Red dash-dotdotted line: nightside ionospheric production rate (left-hand $y$ scale).

Table 2. Ionospheric parameters.

\begin{tabular}{lcc}
\hline \hline Parameter & Symbol & Value \\
\hline Initial ion temperature & $T_{\mathrm{i}}$ & $3000 \mathrm{~K}$ \\
Initial electron temperature & $T_{\mathrm{e}}$ & $3000 \mathrm{~K}$ \\
Absorption cross section & $\sigma_{\mathrm{a}}$ & $6.55 \times 10^{-22} \mathrm{~m}^{-2}$ \\
Ionization cross section & $\sigma_{\mathrm{i}}$ & $6.51 \times 10^{-22} \mathrm{~m}^{-2}$ \\
Ionization rate at infinity & $I_{\infty} \sigma_{\mathrm{i}}$ & $6.29 \times 10^{-6} \mathrm{~s}^{-1}$ \\
Total production rate & $Q_{\mathrm{O}^{+}, \text {tot }}$ & $2.81 \times 10^{29} \mathrm{~s}^{-1}$ \\
Neutral oxygen boundary density & $n_{\mathrm{O} \text {, surf }}$ & $5.00 \times 10^{13} \mathrm{~m}^{-3}$ \\
Neutral oxygen temperature & $T_{\mathrm{O}}$ & $1764 \mathrm{~K}$ \\
Neutral oxygen scale height & $H$ & $92.8 \mathrm{~km}$ \\
\hline
\end{tabular}

and (7) from diverging due to low nightside plasma density. After this is added, the constant background ionization rate is

$Q_{\mathrm{O}^{+}, \text {background }}=0.10 \cdot Q_{\mathrm{O}^{+}}\left(h, 30^{\circ}\right)$.

The ionospheric plasma itself is produced with initial ion and electron temperatures $T_{\mathrm{i}}=T_{\mathrm{e}}=3000 \mathrm{~K}$ similar to that of the upper ionosphere of Venus (Schunk \& Nagy 2000). All ionospheric parameter values are summarized in Table 2. In addition, Fig. 3 shows the neutral profile and the substellar and nightside ion production profiles. As can be seen, the ionization profile is almost proportional to the neutral density, implying that photoabsorption is in practice negligible above the absorbing boundary and that use of a Chapman profile, Eq. (10), although correct is really more sophisticated than necessary.

\section{Results}

The results are illustrated in Figs. $4-8$. Figure 4 is a threedimensional overview of the stellar wind density that compares the perpendicular and the quasiparallel runs, while Figs. 5-8 show miscellaneous physical quantities plotted on the crosssections of the simulation box. Plasma loss rates are summed up in Table 3.

Figures 5-7 are organized such that the left, middle, and right columns of plots refer to the perpendicular, intermediate, and quasiparallel runs, respectively. The three plots in every row show the same quantity. Figure 8 only shows results for the quasiparallel run. The plots contained within each single figure depict the same cross-section in the simulation box. Color scales are kept the same for every physical quantity. For vector plots, the color represents the true magnitude of the three-dimensional vector. The arrow lengths are proportional to the square root of
Table 3. Rate of ionospheric plasma being removed at the outer simulation box boundaries and stellar wind plasma being removed at the planet surface.

\begin{tabular}{lcc}
\hline \hline Simulation Run & $\begin{array}{c}\text { Ionosphere } \\
\left(\mathrm{s}^{-1}\right)\end{array}$ & $\begin{array}{c}\text { Stellar wind } \\
\left(\mathrm{s}^{-1}\right)\end{array}$ \\
\hline Perpendicular run $\left(\alpha_{\mathrm{sw}, 0}=90^{\circ}\right)$ & $1.3 \times 10^{29}(46 \%)$ & $8.1 \times 10^{24}$ \\
Intermediate run $\left(\alpha_{\mathrm{sw}, 0}=30^{\circ}\right)$ & $1.2 \times 10^{29}(42 \%)$ & $9.0 \times 10^{24}$ \\
Quasiparallel run $\left(\alpha_{\mathrm{sw}, 0}=10^{\circ}\right)$ & $9.5 \times 10^{28}(34 \%)$ & $5.8 \times 10^{25}$ \\
\hline
\end{tabular}

Notes. Percentages within brackets refer to the equivalent fraction of the total ionospheric production rate $Q_{\text {tot }}$ in Table 2 . Plasma can only be removed at the planet surface and the outer simulation box boundaries. The numbers are time averages for the quasistationary state at which the plasma production rate of each species equals the respective timeaveraged plasma removal rates (planetary surface plus simulation box boundaries).

the strength of the respective vector quantities projected onto the cross section. The proportionality constants are however not the same in different plots and the arrows are therefore not directly comparable between simulation runs. All quantities are normalized using the equivalent background stellar-wind values.

We begin by looking at the reference run, i.e. the perpendicular run. As anticipated in Sect. 4, the equatorial cross-sections for the perpendicular run in Fig. 5 are all very close to being mirror symmetric whereas the equivalent polar plane cross-sections for the same run in Fig. 6 are not.

The stellar wind density in Figs. $5 \mathrm{~g}$ and $6 \mathrm{~g}$ allows us to identify all the generic features we associate with the interaction between supersonic stellar wind and a conducting obstacle (ionosphere) without an intrinsic magnetic field. The stellar wind enters the simulation box on the negative $x$ side and travels in the positive $x$ direction until it passes through the bow shock, the paraboloid surface surrounding the planet on the dayside and visible as a sudden jump in stellar wind density by a factor of three. Immediately downstream of the bow shock is the magnetosheath where the stellar wind flow is diverted around the planet. Between the magnetosheath and the planet, we find the ionospheric plasma that constitutes the true obstacle because of its conductivity and thermal pressure, Figs. $5 \mathrm{~d}$ and $6 \mathrm{~d}$. The ionosphere is separated from the magnetosheath by the so-called ion composition boundary (ICB).

The basic behavior of the frozen-in magnetic field similarly follows the stellar wind. Although somewhat difficult to see with our color scale it is enhanced by a factor of three over the bow shock in Fig. 5a as the stellar wind is compressed by the same factor. The comoving magnetic field lines subsequently drape the planet as the stellar wind is diverted around it, leading to a dayside pile-up of field lines and a strongly enhanced field strength. Further downstream, this leads to the creation of two lobes with oppositely directed magnetic fields, which are also separated by a current sheet.

Having familiarized ourselves with the perpendicular run, we turn our attention to how the features described above change as the angle $\alpha_{\mathrm{sw}, 0}$ decreases, i.e. as we go to the intermediate run and finally to the quasiparallel run. Looking at the bow shock in primarily Figs. $5 \mathrm{~g}-\mathrm{i}$, and secondarily Figs. $6 \mathrm{~g}-\mathrm{i}$, we can see how a region with a very unstable and not very well-defined quasiparallel shock moves in from the negative $y$ boundary and wanders toward the substellar point. This manifest quasiparallel shock region is, as anticipated, far from a single point but instead corresponds approximately to local shock angles $\theta_{B n}<15^{\circ}$. This also fits with the observation that the quasiparallel shock is visible in 
a) $n_{\mathrm{sw}} / n_{\mathrm{sw}, 0}$

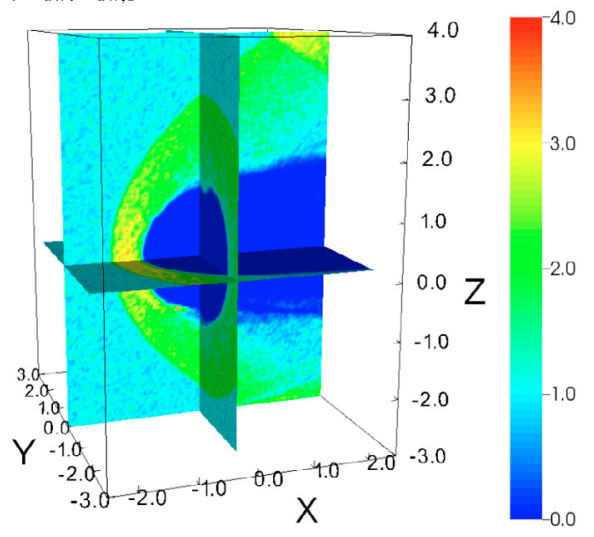

b) $n_{\mathrm{sw}} / n_{\mathrm{sw}, 0}$

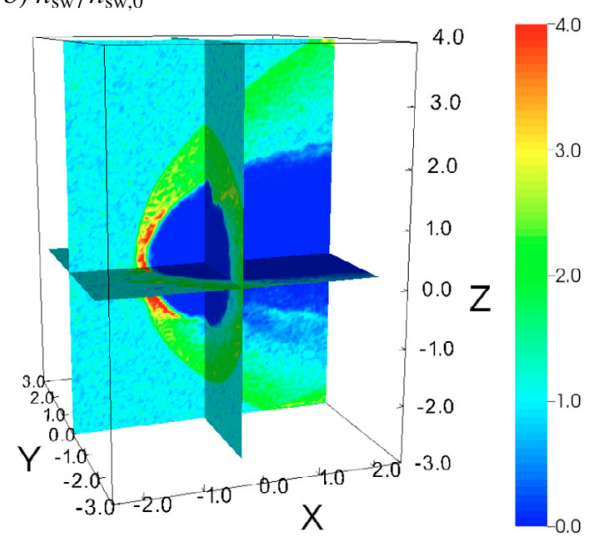

Fig. 4. Three-dimensional overviews of simulation results for the stellar wind density in the form of intersecting cross-sections for the perpendicular a) and quasiparallel b) simulation run. The density is normalized to the background stellar wind density. Parts of the simulation box have been removed to give a clearer overview. The $x, y$, and $z$ axes are in units of $R_{\mathrm{p}}$. the polar plane in the quasiparallel run, Fig. 6i. The transition from quasiperpendicular to quasiparallel shock is also visible in the stellar wind velocity, Figs. $5 j-1$. There we can see that the stellar wind begins to decelerate up to an entire $\sim R_{\mathrm{p}}$ upstream of the original shock surface illustrating both the extent of the quasiparallel foreshock and how the influence of the obstacle then reaches much farther upstream than for the perpendicular run. This extended, and "thick" quasiparallel shock region described above, effectively implies that there is no clear boundary between the undisturbed upstream stellar wind and the downstream magnetosheath, which in the process has become less homogeneous.

The draping of the magnetic field in Figs. $5 \mathrm{a}-\mathrm{c}$ and $6 \mathrm{a}-\mathrm{c}$ predictably becomes weaker as the perpendicular IMF component $B_{y}=B_{\mathrm{sw}, 0} \sin \alpha_{\mathrm{sw}, 0}$ decreases. Furthermore, a low $\alpha_{\mathrm{sw}, 0}$ implies that the draped field lines will form not just a "U" curve around the planet but a kind of "S" curve since they have to connect to the field lines of the IMF. This effect manifests itself in the equatorial plane as the appearance of an irregularly shaped second current sheet in the intermediate and quasiparallel runs besides the familiar current sheet downstream of the planet separating the nightside magnetic lobes in all three runs.

We expect that oxygen ions that for one reason or another find themselves inside the stellar-wind-dominated flow on the southern hemisphere $(z<0)$ are accelerated away from the planet by the convective electric field (Figs. $7 \mathrm{a}-\mathrm{c}$ ) until the $\boldsymbol{v}_{\mathrm{O}^{+}} \times \boldsymbol{B}$ term in the Lorentz force starts to dominate over the electric field and diverts the ions. In an ideal, perfectly homogeneous stellar wind, this pick-up motion results in cycloid-shaped trajectories transporting the ions downstream. The length scale for each cycloid in a perpendicular interaction is the ion gyration radius

$r_{\mathrm{g}, \mathrm{O}^{+}}=\frac{16 m_{\mathrm{p}} v_{\mathrm{sw}, 0}}{e B_{\mathrm{sw}, 0}} \approx 0.72 R_{\mathrm{p}}$,

where $v_{\mathrm{sw}, 0}$ and $B_{\mathrm{sw}, 0}$ are the undisturbed stellar wind velocity and magnetic field strength. Since $r_{\mathrm{g}, \mathrm{O}^{+}}$is of the same order as the size of the planet, this described motion should be able to transport oxygen away in the negative $z$ direction on similar length scales, i.e. we should naively expect an extended and visible pick-up region. Looking at the ionospheric density and velocity in Figs. 6d-f and 7d-f does however tell us that it is not as simple as that. We see a small population of high-speed oxygen ions scattered above the south pole ionosphere and downstream of it. This can be interpreted as the aforementioned pick-up region. It is essentially absent in the perpendicular run but becomes more and more apparent in the intermediate and quasiparallel run, although is still weak. The selected trajectories plotted in Figs. 6d-f support the notion that ions are indeed picked up from the ionosphere.

The absence of (large-scale) pick-up in the perpendicular run is explained by the asymmetric draping which has led to the ionosphere being wrapped in a locally very strong magnetic field as shown in Fig. 6a. The magnetic field is $\sim 20$ times stronger than the background magnetic field implying that the local gyration scale, Eq. (12), is as many times smaller or about $r_{\mathrm{g}, \mathrm{O}^{+}} \sim 0.035 R_{\mathrm{p}}$, which is smaller than the thickness $\sim 0.2 R_{\mathrm{p}}$ of the layer of locally enhanced magnetic field. This means that a cycloidal motion that begins within this layer will generally remain within it. Since the ionospheric production rate profile in practice decreases exponentially with a scale height of $H=0.015 R_{\mathrm{p}}$, we know that almost no ionospheric production will take place outside the enhanced magnetic field, hence there will be no significant visible cycloidal motion. This absence can be compared with the clear presence of an extended pick-up region in e.g. Boesswetter et al. (2004) or Simon et al. (2006); in both of these cases, there is a lack of the same strong and localized magnetic pile-up but also of additional "hot population" exosphere components $Q \propto \ln \left(R_{\mathrm{p}} / r\right)$ producing ions on greater altitude scales, thus ions that are very amenable to being picked up. Upper atmospheres do indeed often have components with higher temperatures and thus greater scale heights. We have however chosen not to include such an extra atmospheric component to reduce the number of arbitrary parameters in our model. An additional consequence of this is the absence of mass loading at altitudes much greater than $H=0.015 R_{\mathrm{p}} \ll R_{\mathrm{p}}$.

When the angle of the stellar-wind magnetic field decreases, the local magnetic field enhancement decreases as $\sim \sin \alpha_{\mathrm{sw}, 0}$ from a maximum of $28 B_{\mathrm{sw}, 0}$ in the perpendicular run to a maximum of $4.2 B_{\mathrm{sw}, 0}$ in the quasiparallel run and in the process the obstacle to large-scale, cycloidal motion disappears. The true motion associated with pick-up is also a bit more complicated than the ideal cycloid motion previously described since 1) the strength of the convective electric field, $\boldsymbol{E}=-\boldsymbol{v}_{\mathrm{sw}} \times \boldsymbol{B}$, that initially accelerates the ions depends on $\alpha_{\mathrm{sw}, 0} ; 2$ ) the direction of the cycloid motion also depends on $\alpha_{\mathrm{sw}, 0}$ implying that the ion trajectories lead not only downstream but also sideways; and 3) the trajectories cannot be ideal cycloids since the enhanced magnetic field has a strong curvature around the south pole due to draping, although this cannot be clearly seen in the included cross sections. Figures $8 \mathrm{a}$ and b clearly illustrate that pickedup ions in the quasiparallel run gyrate not only downstream as would be expected in an ideal perpendicular situation but also sideways. This is also likely the origin of the few particles of 
a) $\boldsymbol{B} / B_{\mathrm{sw}, 0}$

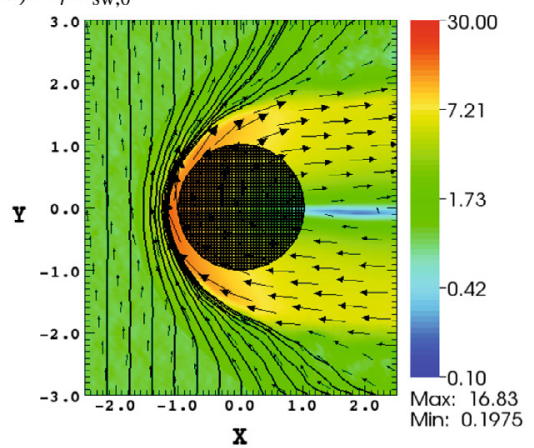

d) $n_{\mathrm{is}} / n_{\mathrm{sw}, 0}$

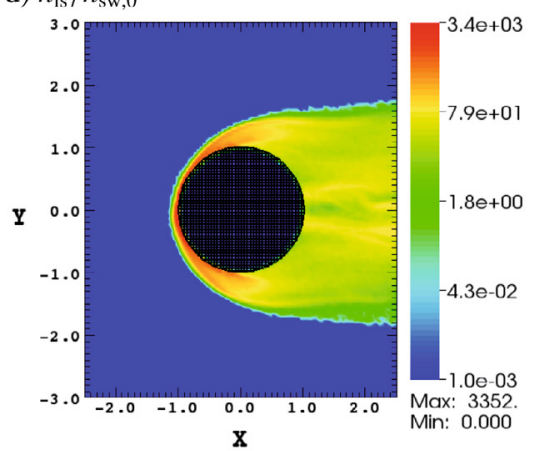

g) $n_{\mathrm{sw}} / n_{\mathrm{sw}, 0}$

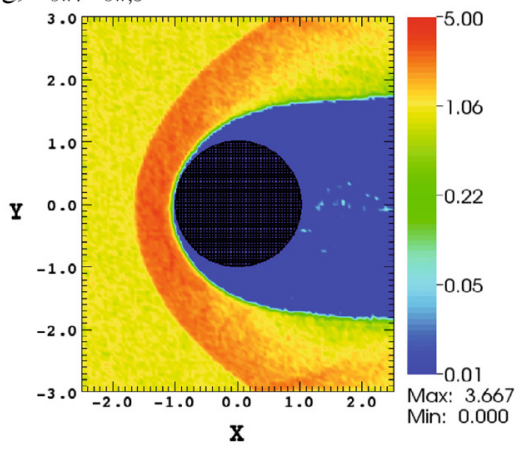

j) $\boldsymbol{v}_{\mathrm{sw}} / v_{\mathrm{sw}, 0}$

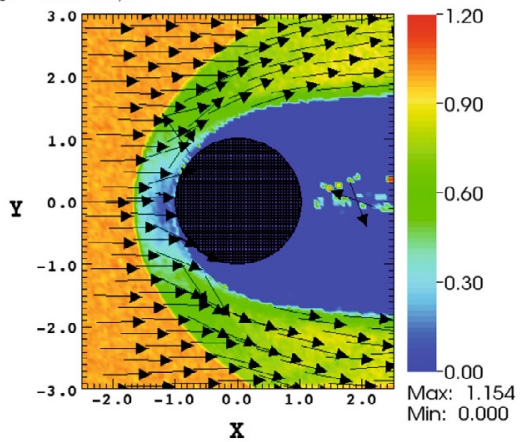

b) $\boldsymbol{B} / B_{\mathrm{sw}, 0}$

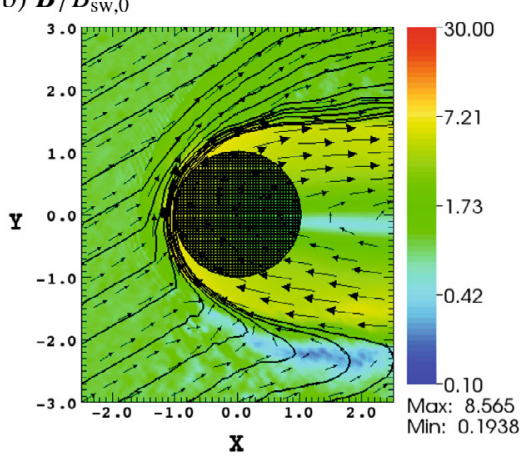

e) $n_{\mathrm{is}} / n_{\mathrm{sw}, 0}$

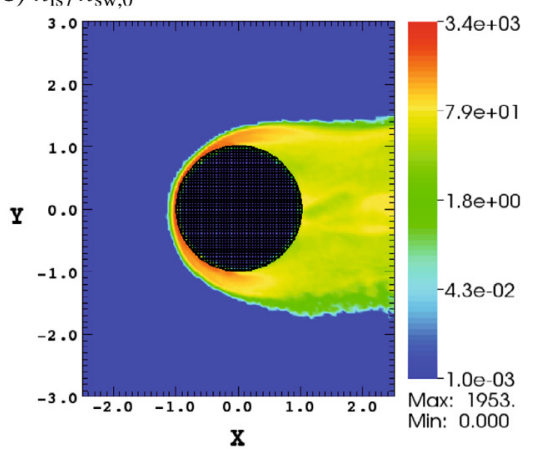

h) $n_{\mathrm{sw}} / n_{\mathrm{sw}, 0}$

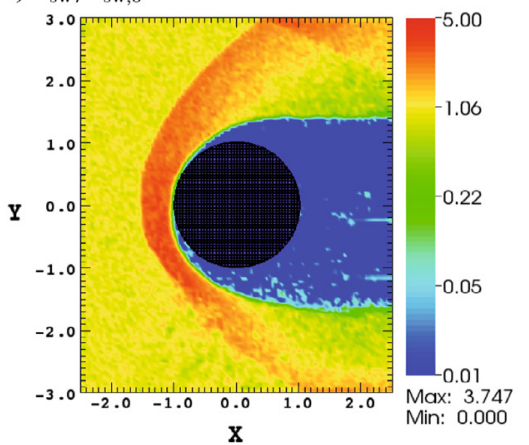

k) $v_{\mathrm{sw}} / v_{\mathrm{sw}, 0}$

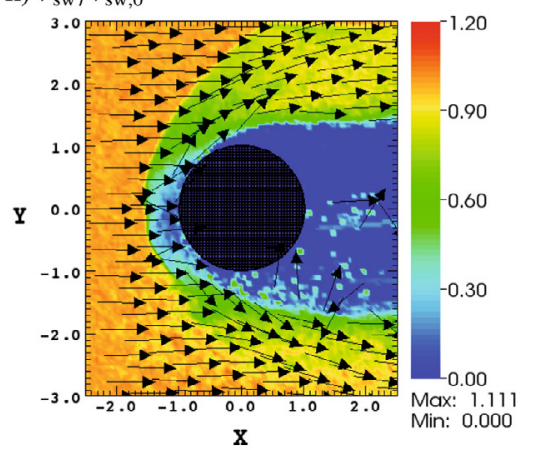

c) $\boldsymbol{B} / B_{\mathrm{sw}, 0}$

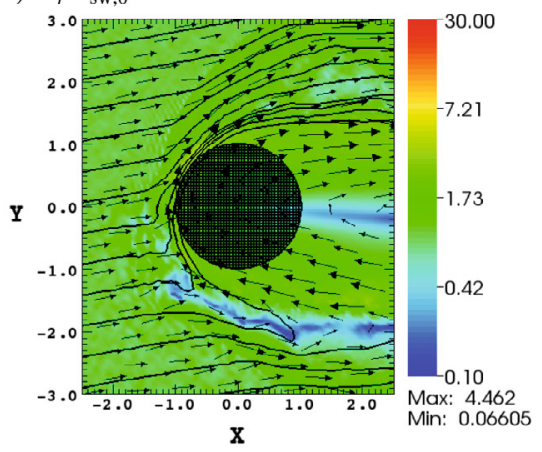

f) $n_{\mathrm{is}} / n_{\mathrm{sw}, 0}$

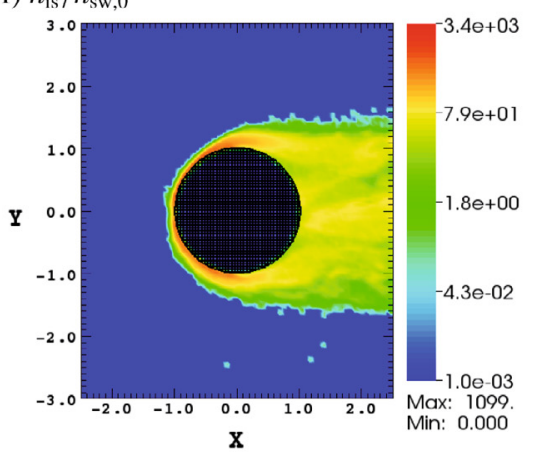

i) $n_{\mathrm{sw}} / n_{\mathrm{sw}, 0}$

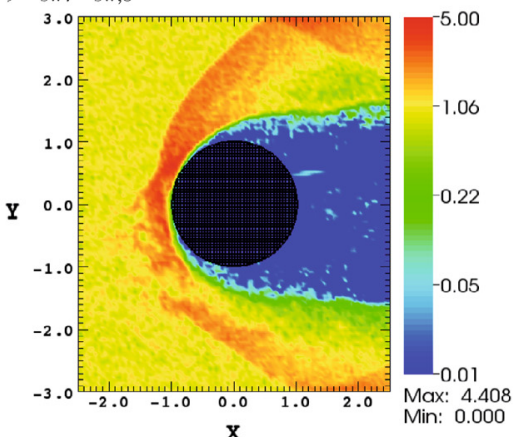

1) $v_{\mathrm{sw}} / v_{\mathrm{sw}, 0}$

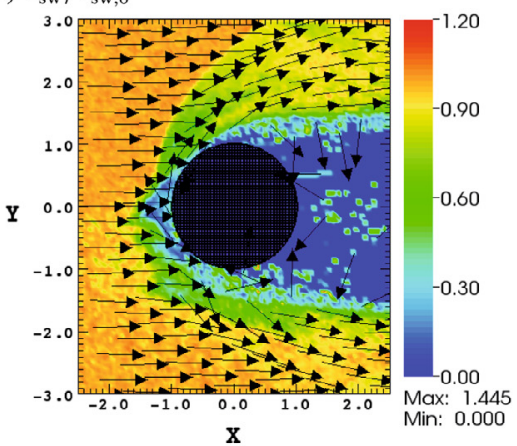

Fig. 5. Simulation results in the form of equatorial plane cross-sections for the perpendicular (left column), intermediate (middle column), and quasiparallel (right column) simulation run. The first row $\mathbf{a}-\mathbf{c}$ ) shows the magnetic field, the second row $\mathbf{d}-\mathbf{f}$ ) the ionospheric density, the third row $\mathbf{g}-\mathbf{i})$ the stellar wind density, and the fourth row $\mathbf{j}-\mathbf{l}$ ) the stellar wind velocity. The $x$ and $y$ axes are in units of $R_{\mathrm{p}}$. A selection of magnetic field lines have been added to $\mathbf{a}-\mathbf{c}$ ).

stray oxygen well outside the main ionosphere in the equatorial plane in Fig. 5f.

The location of the ICB and the extent of the ionosphere in the polar plane is more difficult to explain, in particular why it consistently runs so close to the planetary surface around the south pole as can be seen in Figs. $6 \mathrm{~d}-\mathrm{f}$ and $6 \mathrm{~g}-\mathrm{i}$. Combining this with Fig. 8a indicates that this phenomenon is very local to the polar cross-section. This does not however contradict the notion of pick-up, which only affects ionospheric ions that appear on the stellar-wind-dominated side of the ICB.

Turning our attention to the densities in the equatorial plane, Figs. $5 \mathrm{~d}-\mathrm{f}$ and $5 \mathrm{~g}-\mathrm{i}$, we can note how the ICB more or less follows the draped magnetic field lines in the equatorial plane and becomes less and less well-defined on the flanks as the 
a) $\boldsymbol{B} / B_{\mathrm{sw}, 0}$

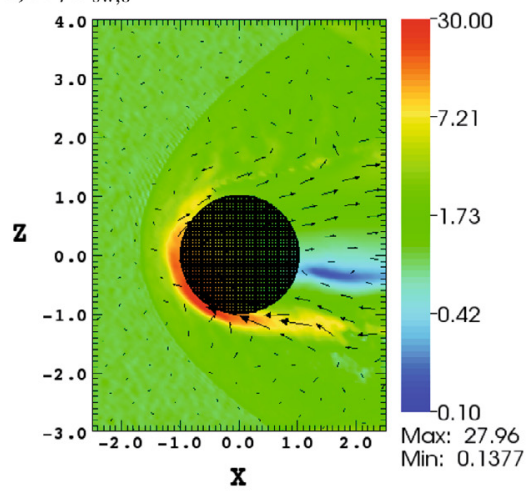

d) $n_{\text {is }} / n_{\mathrm{sw}, 0}$

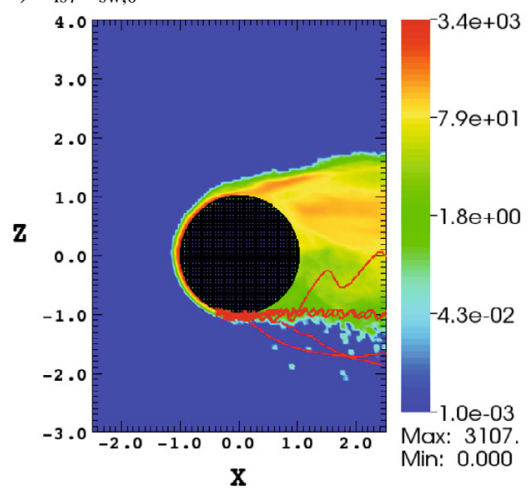

g) $n_{\mathrm{sw}} / n_{\mathrm{sw}, 0}$

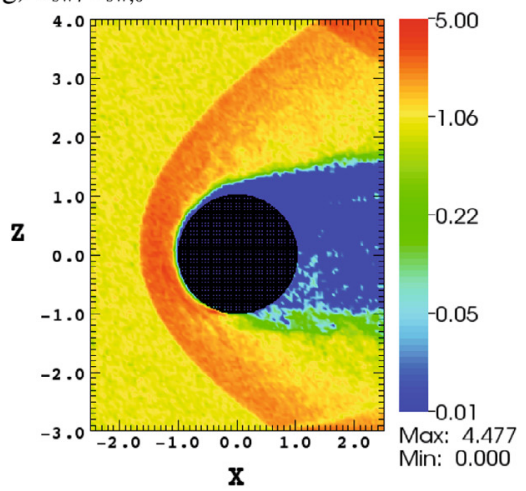

b) $\boldsymbol{B} / B_{\mathrm{sw}, 0}$

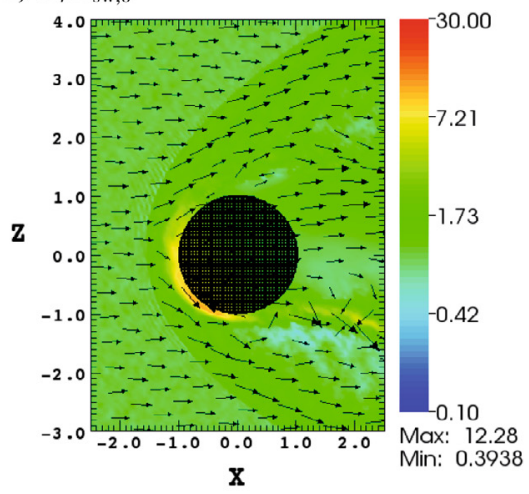

e) $n_{\text {is }} / n_{\text {sw, } 0}$

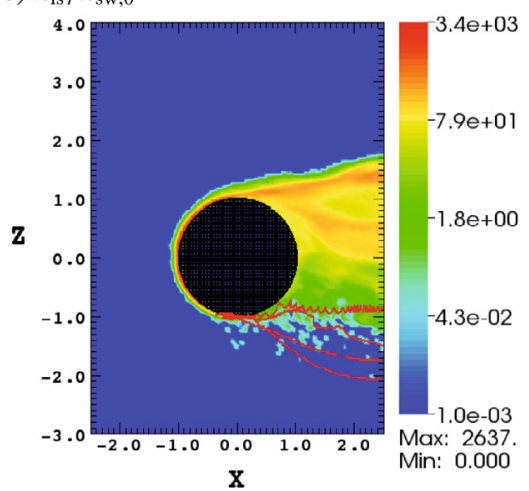

h) $n_{\mathrm{sw}} / n_{\mathrm{sw}, 0}$

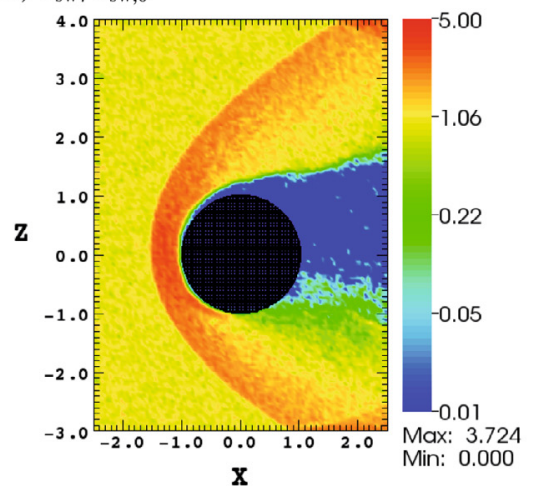

c) $\boldsymbol{B} / B_{\mathrm{sw}, 0}$

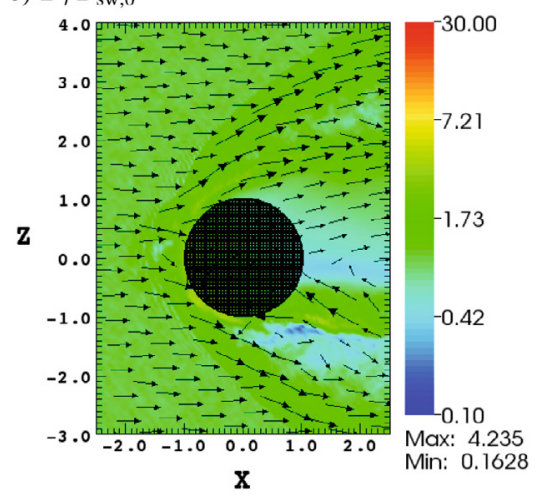

f) $n_{\text {is }} / n_{\mathrm{sw}, 0}$

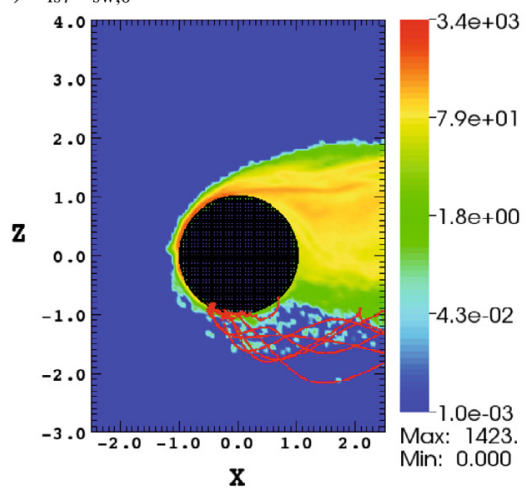

i) $n_{\mathrm{sw}} / n_{\mathrm{sw}, 0}$

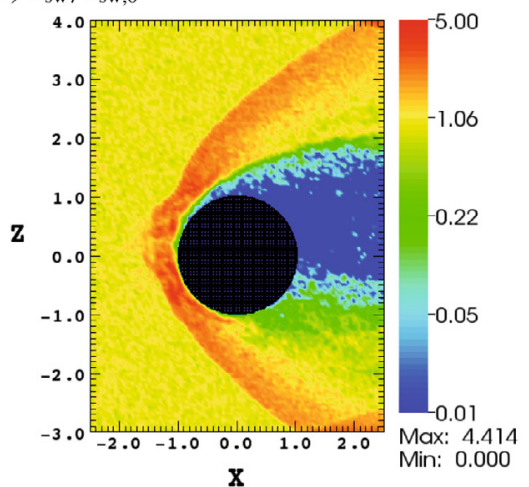

Fig. 6. Simulation results in the form of polar plane cross-sections for the perpendicular (left column), intermediate (middle column), and quasiparallel (right column) simulation run. The first row $\mathbf{a}-\mathbf{c}$ ) shows the magnetic field, the second row $\mathbf{d}-\mathbf{f}$ ) the ionospheric density, and the third row g-i) the stellar wind density. The $x$ and $z$ axes are in units of $R_{\mathrm{p}}$. A selection of ion trajectories (red) originating in the higher south pole ionosphere and projected on to the polar plane have been added to $\mathbf{d}-\mathbf{f}$ ). The trajectories in f) are identical to those in Fig. $8 \mathrm{a}$.

weakening magnetic field becomes unable to keep the two species from diffusing into each other. It is difficult to discern from the cross-sections but still unambiguous in both the equatorial and the polar plane that the stellar wind progressively reaches closer and closer to the dayside planet surface for lower values of $\alpha_{\mathrm{sw}, 0}$. This implies that $\alpha_{\mathrm{sw}, 0}$ may influence the extent to which the stellar wind plasma is lost to the planet if any. The statistics for time-averaged plasma losses are summed up in Table 3. Although this is indeed a very small data set we note that when $\alpha_{\mathrm{sw}, 0}$ decreases ionospheric plasma loss from the planet also decreases, first by $8 \%$ and then by another $21 \%$. At the same time, stellar wind loss to the planet increases first by $11 \%$ and then by $640 \%$. Thus, both trends seem to accelerate for lower values of $\alpha_{\mathrm{sw}, 0}$. The highest stellar wind loss to the planet, $5.8 \times 10^{25} \mathrm{~s}^{-1}$, is however still small compared to both ionospheric losses, $\sim 10^{29} \mathrm{~s}^{-1}$, and the stellar wind flow as measured by the flow through a planetary cross-section, $n_{\mathrm{sw}, 0} v_{\mathrm{sw}, 0} \pi R_{\mathrm{p}}{ }^{2} \sim$ $10^{28} \mathrm{~s}^{-1}$.

\section{Discussion and conclusions}

The existence of exoplanets with orbits smaller than that of Mercury implies that some of these are experiencing a stellar wind in which the IMF is parallel to the stellar wind direction. Since many of the generic features of stellar wind interaction depend on the existence of a non-zero perpendicular IMF component, one can expect these interactions to result in very different types of magnetospheres. We have therefore chosen to study the importance of quasiparallel stellar-wind interaction by using a 3D plasma hybrid simulation model applied to the Venus-like interaction between a stellar wind and a terrestrial, unmagnetized 
a) $\boldsymbol{E} /\left|v_{\mathrm{sw}, 0} B_{\mathrm{sw}, 0}\right|$

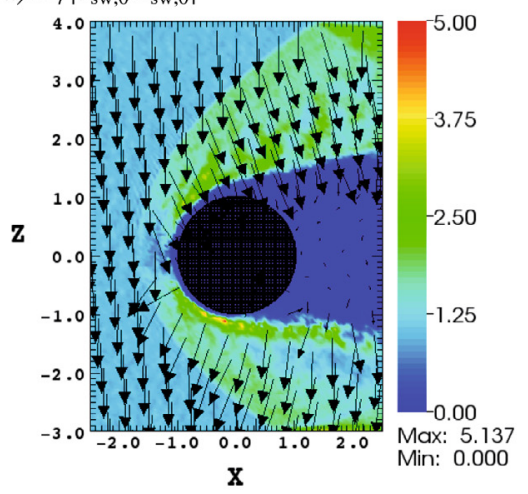

d) $v_{\text {is }} / v_{\mathrm{sw}, 0}$

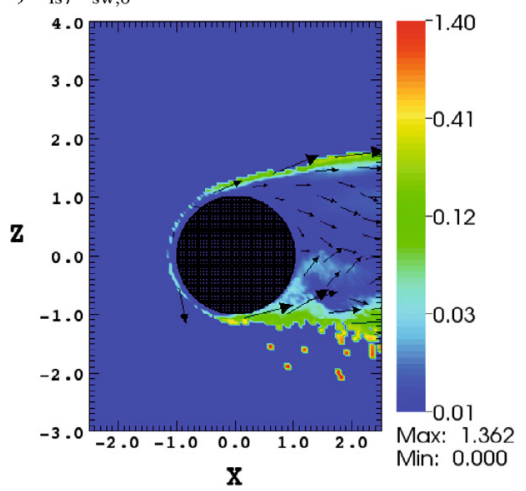

b) $\boldsymbol{E} /\left|v_{\mathrm{sw}, 0} B_{\mathrm{sw}, 0}\right|$

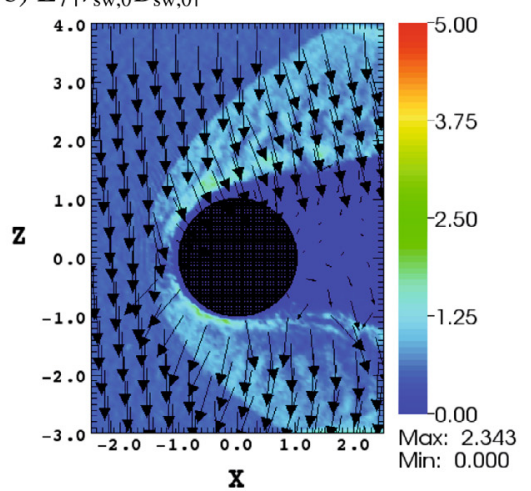

e) $v_{\text {is }} / v_{\mathrm{sw}, 0}$

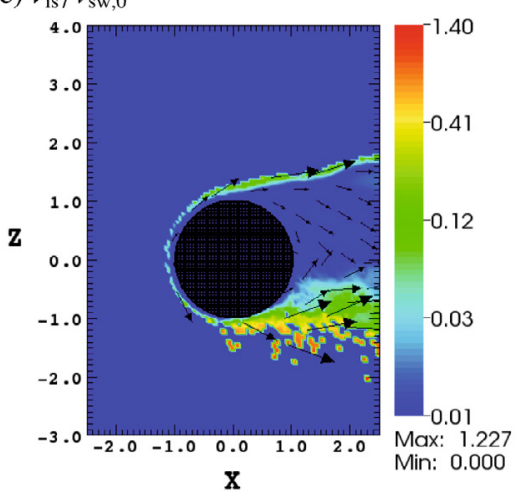

c) $\boldsymbol{E} /\left|v_{\mathrm{sw}, 0} B_{\mathrm{sw}, 0}\right|$

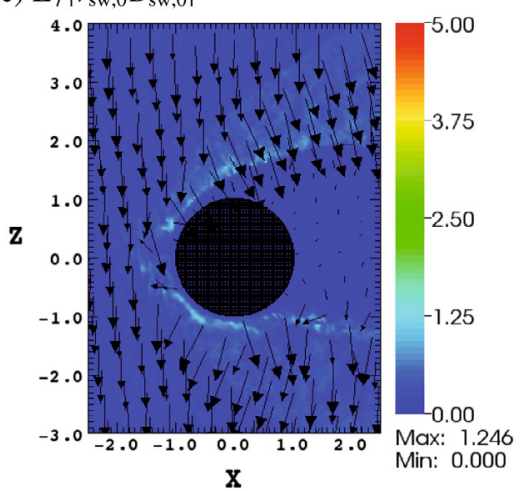

f) $\boldsymbol{v}_{\mathrm{is}} / v_{\mathrm{sw}, 0}$

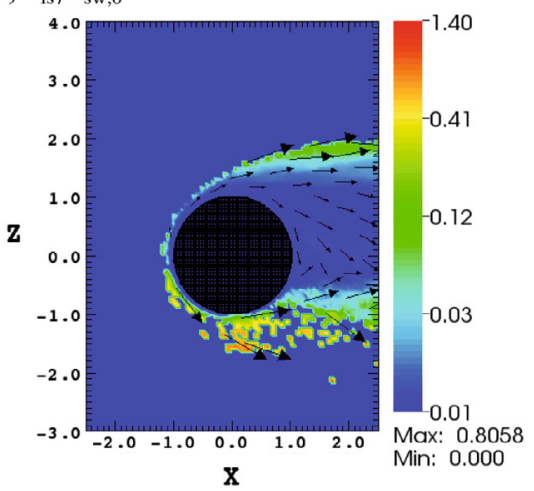

Fig. 7. Simulation results in the form of polar plane cross-sections for the perpendicular (left column), intermediate (middle column), and quasiparallel (right column) simulation run. The first row $\mathbf{a}-\mathbf{c}$ ) shows the electric field and the second row $\mathbf{d}-\mathbf{f}$ ) the stellar wind velocity. The $x$ and $z$ axes are in units of $R_{\mathrm{p}}$.

a) $n_{\mathrm{is}} / n_{\mathrm{sw}, 0}$

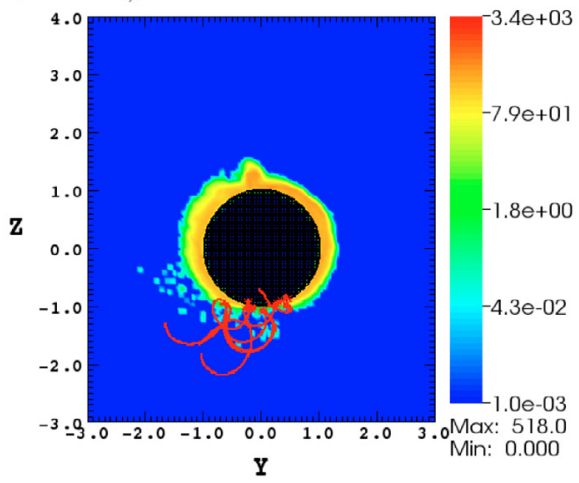

b) $\boldsymbol{v}_{\text {is }} / v_{\mathrm{sw}, 0}$

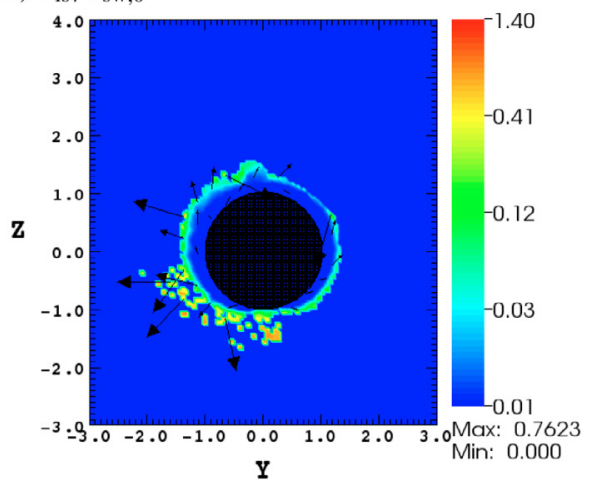

Fig. 8. Simulation results in the form of terminator cross-sections for the quasiparallel run. Figure a) (left) is the ionospheric density and b) (right) is the ionospheric velocity. The $y$ and $z$ axes are in units of $R_{\mathrm{p}}$. A selection of ion trajectories (red) originating in the higher south pole ionosphere and projected on to the terminator plane have been added to a). The same trajectories have been added to Fig. 6f.
Earth-sized planet equipped with an ionosphere and orbiting a Sun-like star at 0.2 AU. We have then compared three simulation runs, all identical with the exception of the angle between IMF and stellar wind velocity.

As expected, we have observed several significant quantitative and qualitative variations by changing the IMF-stellar wind angle from $90^{\circ}$ to $30^{\circ}$, and finally to $10^{\circ}$. The main changes we observe are that 1) a large part of the substellar bow shock is replaced by a vaguely defined and unstable quasiparallel shock; 2) magnetic draping and pile-up are greatly weakened; 3) a second, very irregular current sheet is created next to the planet due to the need for the IMF field lines to connect with the magnetic lobe antiparallel to the IMF; 4) the pick-up increases due to weakening (asymmetric) magnetic pile-up; 5) dayside ICB moves closer to the absorbing planet surface; and 6) less ionospheric plasma is lost from the planet and more stellar wind is lost to the planet.

It is difficult to argue against all of 1)-3) being present in some form for (supersonic) quasiparallel stellar wind interactions with conducting obstacles. Not too many conclusions can be drawn from 4), the increasing pick-up, since it should depend on our exact choice of parameters and in particular on us working in a low magnetic field limit.

We briefly consider how these results can be generalized to smaller IMF-stellar wind angles. Before doing so, it is worth pointing out that both the second current sheet and the increasing pick-up are only transitional and should for obvious reasons ultimately disappear for a perfectly parallel interaction. The quasiparallel bow shock should continue to move towards the substellar point for decreasing angles and the magnetic draping and 
lobes should continue to weaken and disappear. The final magnetosphere for a parallel interaction should theoretically be cylindrically symmetric, assuming that it is always stable and laminar, which indicates the great differences one expects between our quasiparallel run and a parallel run.

The trend that the ICB and the stellar wind reaches closer and closer to the dayside absorbing planetary surface for smaller and smaller IMF-stellar wind angles is corroborated by our experience from similar quasiparallel and parallel test runs not presented in this work. In those runs, we have more often than not encountered the phenomenon that stellar wind reaches through the ionosphere and impacts the absorbing planetary surface in bulk for IMF-stellar wind angles smaller than the $10^{\circ}$ in our quasiparallel run. As previously mentioned, this effect is one of the reasons why we have not formally included such a simulation run in our study since it needs a more careful treatment of the chemistry between stellar wind and ionosphere, which we have not yet modeled.

This relates to the observation that the amount of stellar wind being lost to the planet, Table 3 , increases when the angle decreases and that this trend seems to accelerate for low angles. Therefore, one may suspect that the amount of stellar wind being absorbed by the planet or interacting with the atmosphere is even greater and more sensitive to the IMF-stellar wind angle at smaller angles. The more sensitive the configuration is to the exact angle, the more incorrect it is to consider in terms of an exoplanet's average interaction over, say, an elliptical orbit or over shifting types of stellar wind (slow/fast). Therefore, future study should if possible not only compare results for several small angles and if necessary adequately handle reactions between stellar wind and atmosphere, but perhaps incorporate knowledge of the time variation in the IMF-stellar-wind angle. If one assumes this angle dependance to hold true in general for stellar wind interaction with unmagnetized planets with atmospheres, then one may speculate that there might be cases where the increased direct interaction between stellar wind and atmosphere because of the low IMF-stellar-wind angle produces higher amounts of energetic neutral atoms (ENAs). These ENAs could then in principle be observable in future high resolution transit spectra in analogy with some interpretations of the Ly $\alpha$ observations of HD 209458 b (Holmström et al. 2008; Ben-Jaffel \& Sona Hosseini 2010).

As mentioned, our simulations also indicate that atmospheric mass loss varies with the IMF-stellar-wind angle, especially at low angles. We infer this directly from the counting of superparticles but in principle also indirectly, although without quantifying it, as a byproduct of the atmospheric ion chemistry (e.g. charge exchange, impact ionization, which are not included in our simulation model) due to stellar wind penetrating deeper into the actual atmosphere with decreasing angle. If we also assume this angle dependance to hold true in general, then the IMFstellar wind angle might also be important when relating observations of exoplanetary atmospheres to planet formation, should the mass loss influence planetary evolution. Mass loss estimates, including considerations of influence on planetary evolution, are already an active topic for cEGPs (see e.g. Yelle 2004; Yelle et al. 2008; Vidal-Madjar et al. 2003, 2008; Tian et al. 2005; Lecavelier des Etangs et al. 2004; Penz et al. 2008; Baraffe et al. 2004; Ben-Jaffel 2007).
Acknowledgements. The authors acknowledge the fellowship of E.P.G.J. from the International Max Planck Research School (IMPRS) on Physical Processes in the Solar System and Beyond of the Max Planck Institute for Solar System Research (MPS) and the Universities of Braunschweig and Göttingen. The Deutsche Forschungsgemeinschaft supported the work of E.P.G.J. partly through grant MO539/15 and of J.M. through grant MO539/16.

\section{References}

Bagdonat, T., \& Motschmann, U. 2002, J. Comp. Phys., 183, 470

Baraffe, I., Selsis, F., Chabrier, G., et al. 2004, A\&A, 419, L13

Baumjohann, W., \& Treumann, R. A. 1996, Basic space plasma physics (London: Imperial College Press)

Ben-Jaffel, L. 2007, ApJ, 671, L61

Ben-Jaffel, L., \& Sona Hosseini, S. 2010, ApJ, 709, 1284

Boesswetter, A., Bagdonat, T., Motschmann, U., \& Sauer, K. 2004, Annales Geophysicae, 22, 4363

Bordé, P., Rouan, D., \& Léger, A. 2003, A\&A, 405, 1137

Borucki, W. J., Koch, D., Basri, G., et al. 2010, Science, 327, 977

Burgess, D. 1989, Geophys. Res. Lett., 16, 345

Chamberlain, J. W., \& Hunten, D. M. 1987, Theory of planetary atmospheres, An introduction to their physics andchemistry, ed. J. W. Chamberlain, \& D. M. Hunten

Farrell, W. M., Desch, M. D., \& Zarka, P. 1999, J. Geophys. Res., 104, 14025

Filippychev, D. S. 2000, Comput. Math. Mod., 11, 15

Fogg, M. J., \& Nelson, R. P. 2009, A\&A, 498, 575

Gaidos, E., Haghighipour, N., Agol, E., et al. 2007, Science, 318, 210

Griessmeier, J.-M., Preusse, S., Khodachenko, M., et al. 2007a, Planet. Space Sci., 55, 618

Griessmeier, J.-M., Zarka, P., \& Spreeuw, H. 2007b, A\&A, 475, 359

Holmström, M., Ekenbäck, A., Selsis, F., et al. 2008, Nature, 451, 970

Huebner, W. F., Keady, J. J., \& Lyon, S. P. 1992, Ap\&SS, 195, 1

Ip, W.-H., Kopp, A., \& Hu, J.-H. 2004, ApJ, 602, L53

Johansson, E. P. G., Bagdonat, T., \& Motschmann, U. 2009, A\&A, 496, 869

Kallio, E., Jarvinen, R., \& Janhunen, P. 2006, Planet. Space Sci., 54, 1472

Lammer, H., Selsis, F., Ribas, I., et al. 2004, in Stellar Structure and Habitable Planet Finding, ed. F. Favata, S. Aigrain, \& A. Wilson, ESA SP-538, 339

Lanza, A. F. 2009, A\&A, 505, 339

Lawson, P. R., Unwin, S. C., \& Beichman, C. A. 2004, Precursor Science for the Terrestrial Planet Finder, Jet Propulsion Laboratory, California Institute of Technology, JPL Publication, 04-014

Lazio, T. J. W., Farrell, W. M., Dietrick, J., et al. 2004, ApJ, 612, 511

Lecavelier des Etangs, A., Vidal-Madjar, A., McConnell, J. C., \& Hébrard, G. 2004, A\&A, 418, L1

Lin, D. N. C. 2006, Overview and prospective in theory and observation of planet formation, ed. H. Klahr, \& W. Brandner (Cambridge University Press), 256

Martinecz, C., Boesswetter, A., Fraenz, M., et al. 2009, J. Geophys. Res. (Space Phys.), 114

Penz, T., Erkaev, N. V., Kulikov, Y. N., et al. 2008, Planet. Space Sci., 56, 1260 Preusse, S., Kopp, A., Büchner, J., \& Motschmann, U. 2005, A\&A, 434, 1191 Raymond, S. N., Mandell, A. M., \& Sigurdsson, S. 2006, Science, 313, 1413

Richards, P. G., Fennelly, J. A., \& Torr, D. G. 1994, J. Geophys. Res., 99, 8981

Schunk, R. W., \& Nagy, A. F. 2000, Ionospheres: Physics, Plasma Physics, and Chemistry (Cambridge University Press)

Simon, S., Boesswetter, A., Bagdonat, T., Motschmann, U., \& Glassmeier, K.-H. 2006, Annales Geophysicae, 24, 1113

Tian, F., Toon, O. B., Pavlov, A. A., \& De Sterck, H. 2005, ApJ, 621, 1049

Treumann, R. A., \& Jaroschek, C. H. 2008a, [arXiv: 0805 . 2162]

Treumann, R. A., \& Jaroschek, C. H. 2008b, [arXiv: 0805. 2579]

Vidal-Madjar, A., Lecavelier des Etangs, A., Désert, J.-M., et al. 2003, Nature, 422, 143

Vidal-Madjar, A., Désert, J.-M., Lecavelier des Etangs, A., et al. 2004, ApJ, 604, L69

Vidal-Madjar, A., Lecavelier des Etangs, A., Désert, J., et al. 2008, ApJ, 676, L57

Yelle, R., Lammer, H., \& Ip, W. 2008, Space Sci. Rev., 139, 437

Yelle, R. V. 2004, Icarus, 170, 167

Zarka, P., Treumann, R. A., Ryabov, B. P., \& Ryabov, V. B. 2001, Ap\&SS, 277, 293 